\title{
Adiabatic renormalization of inflationary perturbations
}

\author{
Ruth Durrer, ${ }^{1, *}$ Giovanni Marozzi, ${ }^{2, \dagger}$ and Massimiliano Rinaldi ${ }^{1, \hbar}$ \\ ${ }^{1}$ Université de Genève, Départment de Physique Théorique, 24 quai Ernest Ansermet Switzerland-1211 Genève 4, Switzerland \\ ${ }^{2}$ Dipartimento di Fisica dell'Universitá di Bologna and Istituto Nazionale Fisica Nucleare, Via Irnerio, 46, 40126 Bologna, Italy
}

(Received 26 June 2009; published 23 September 2009)

\begin{abstract}
We discuss the impact of adiabatic renormalization on the power spectrum of scalar and tensor perturbations from inflation. We show that adiabatic regularization is ambiguous as it leads to very different results, for different adiabatic subtraction schemes, both in the range $v \equiv k /(a H) \gtrsim 0.1$ and in the infrared regime. All of these schemes agree in the far ultraviolet, $v \gg 1$. Therefore, we argue that in the far infrared regime, $v \ll 1$, the adiabatic expansion is no longer valid, and the unrenormalized spectra are the physical, measurable quantities. These findings cast some doubt on the validity of the adiabatic subtraction at horizon exit, $v=1$, to determine the perturbation spectra from inflation which has recently been advocated in the literature.
\end{abstract}

DOI: 10.1103/PhysRevD.80.065024

PACS numbers: 11.10.Gh, 04.62.+v, 98.70.Vc

\section{INTRODUCTION}

Inflation was originally proposed to solve the initial condition problem of standard big bang cosmology. At the same time, it was found that inflation typically leads to a nearly scale invariant spectrum of scalar and tensor fluctuations [1,2]. It is this finding, which is so well confirmed by the observed anisotropies and polarization in the cosmic microwave background [3], which has led to a wide acceptance of the inflationary paradigm. Using present and future $\mathrm{CMB}$ data, in combination with other cosmological data sets, we are now in the position to constrain models.

Typically, an inflationary model predicts the value of three parameters, namely, the scalar spectral index $n_{s}$, the tensor spectral index $n_{t}$, and the tensor-to-scalar ratio $r$. So far, observations just provide upper limits on tensor fluctuations. These are not independent of the scalar spectral index $n_{s}$ as it is evident from the two-dimensional one- and two- $\sigma$ confidence contours, shown in Fig. 5 of Ref. [4]. These data can be used to constrain inflationary models. For example, in [4] it is noted that a model of inflation with a scalar field potential of the form $\lambda \phi^{4}$ is ruled out if the number $N$ of $e$ foldings of inflation after horizon crossing of the scales probed by WMAP, $k \simeq 0.002 h / \mathrm{Mpc} \simeq 6 H_{0}$, is of the order of $N \lesssim 50-60$. In this expression, $H_{0}$ is the current value of the Hubble parameter, and $h=0.72 \pm$ 0.08 .

This is a truly breathtaking result meaning that $\mathrm{CMB}$ data, i.e. cosmological observations on the largest scales, can provide information about the physics at energy scales much higher than those attainable in the laboratory, hence about the physics on the smallest scales. It is therefore of the utmost importance that these results are subjected to the deepest scrutiny. With this point in mind, we have

\footnotetext{
*ruth.durrer@unige.ch

marozzi@bo.infn.it

massimiliano.rinaldi@unige.ch
}

studied the recent works [5-7]. In particular, in Ref. [5], the author argues that the inflationary power spectra, as they are usually calculated, are not correct. In fact, since they diverge at coincident points, one should subtract an appropriate adiabatic counterterm (see also [8] for a different point of view). In Ref. [7] the authors perform explicit calculations along these lines and subtract the adiabatic term at the Hubble exit, namely, when $v=k /(a H)=1$. As a result, the values of the tensor-to-scalar ratio that they find differ significantly from the ones usually adopted to be compared with the data of [4]. The most surprising consequence is that, for example, the chaotic inflationary model $\lambda \phi^{4}$ is no longer ruled out by the WMAP data.

It is well-known that the standard power spectra are nearly time independent on super-Hubble scales, i.e. when $v \leq 1$. In this paper, we show that this is not the case for the adiabatic contribution to the spectra in a realistic model of inflation. The renormalized spectra, no matter what approach is used for the adiabatic subtraction, always depend on $v$. The adiabatic regularization, even when performed at horizon exit or a few Hubble times later, is ambiguous in the sense that it gives a different result depending on which approach is used. In some cases, the result is even strongly time dependent. In fact, we show that there are different ways to perform the adiabatic subtraction, which all agree in the far ultraviolet regime, but yield very different results for $v \leq 1$. The most reasonable adiabatic expression actually yields an adiabatic spectrum $P^{(2)}$ such that the ratio $P^{(2)} / P^{(\mathrm{IR})}$ becomes quickly negligible for $v \ll 1$, indicating that the spectrum is not modified.

These considerations indicate that the correct time at which the adiabatic subtraction has to be performed is the end of inflation, rather than the time of Hubble exit. However, at the end of inflation, all of the modes relevant for observational cosmology are in the far infrared region where the adiabatic expansion seems inappropriate as the expansion of the Universe is not slow compared to the 
oscillation frequency of the mode and, thus, not adiabatic. If one insists, however, to extend the adiabatic regularization in the infrared, we present an argument, which shows that the adiabatic counterterms become negligible anyway. Thus, we shall argue that the physical result is not affected by adiabatic regularization.

The paper is organized as follows: in the next section we present approximate expressions for the scalar and tensor power spectra in the framework of slow-roll inflation. In Sec. III, we discuss different adiabatic subtractions for both the scalar and tensor power spectrum, and we argue that the difference with the original power spectra becomes irrelevant in the far infrared regime. In Sec. IV, we draw our conclusions. Some technical results are deferred to appendices.

\section{POWER SPECTRA FROM SLOW-ROLL INFLATION}

\section{A. Linear perturbations in slow-roll inflation}

We consider a spatially flat Universe, whose dynamics is driven by a classical minimally coupled scalar field, described by the action

$$
S=\int d^{4} x \sqrt{-g}\left[\frac{R}{16 \pi G}-\frac{1}{2} g^{\mu \nu} \partial_{\mu} \phi \partial_{\nu} \phi-V(\phi)\right] .
$$

For a spatially flat Friedmann space-time, of the form $d s^{2}=-d t^{2}+a^{2}(t) \delta_{i j} d x^{i} d x^{j}$, the background equations of motion for $\phi$ and for the scale factor $a(t)$ read

$$
\begin{gathered}
\ddot{\phi}+3 H \dot{\phi}+V_{\phi}=0, \\
\left(\frac{\dot{a}}{a}\right)^{2}=H^{2}=\frac{1}{3 M_{\mathrm{pl}}^{2}}\left[\frac{\dot{\phi}^{2}}{2}+V\right], \\
\dot{H}=-\frac{1}{2 M_{\mathrm{pl}}^{2}} \dot{\phi}^{2},
\end{gathered}
$$

where $M_{\mathrm{pl}}^{2}=1 /(8 \pi G)$ is the reduced Planck mass. The dot denotes a derivative with respect to the cosmic time $t$. Linear perturbations of the metric in longitudinal gauge are given by

$$
d s^{2}=-(1+2 \Psi) d t^{2}+a^{2}\left[(1-2 \Phi) \delta_{i j}+h_{i j}\right] d x^{i} d x^{j} .
$$

Here, $\Psi$ and $\Phi$ represent the Bardeen potentials, and $h_{i j}$ describes traceless, transverse tensor degrees of freedom, that is gravitational waves. We do not discuss vector perturbations.

In single-field inflationary models, and to first order in perturbation theory, we have $\Phi=\Psi$. Scalar perturbations have only one degree of freedom, which can be studied by means of a single gauge invariant variable, such as the socalled Mukhanov variable [9], defined, in longitudinal gauge, by

$$
Q=\varphi+\frac{\dot{\phi}}{H} \Psi .
$$

In this expression, we assume that the scalar field can be written as a background term plus a linear perturbation, namely, as $\phi+\varphi$.

Often, one also uses the curvature variable $\zeta$, which, for $\Phi=\Psi$, is defined as [10]

$$
\zeta=\frac{H}{\dot{\phi}} Q=\frac{2\left(H^{-1} \dot{\Psi}+\Psi\right)}{3(1+w)}+\Psi .
$$

Here, $w$ is the equation of state parameter, which satisfies

$$
1+w=\frac{2 \dot{\phi}^{2}}{\dot{\phi}^{2}+2 V(\phi)} .
$$

It is important to note that both $\zeta$ and $Q$ are related to the Bardeen potential $\Psi$ via a first order equation. They are not independent degrees of freedom, and it is therefore not consistent to think of $Q$ as a quantum degree of freedom and of $\Psi$ as a classical variable. When we quantize $Q$, or rather $a Q$ as below, we also quantize the Bardeen potential. In fact, we do not equate expectation values of some quantum fields to classical first order perturbations of the metric via Einstein's equation, but we do quantize the metric perturbations.

The equation governing $Q$ in Fourier space, is given by

$$
\ddot{Q}_{k}+3 H \dot{Q}_{k}+\frac{1}{a^{2}} k^{2} Q_{k}+\left[V_{\phi \phi}+2 \frac{d}{d t}\left(3 H+\frac{\dot{H}}{H}\right)\right] Q_{k}=0,
$$

where $V_{\phi \phi}$ denotes the second derivative of $V$ with respect to $\phi$. During inflation, we assume that the so-called slowroll parameters

$$
\epsilon=\frac{M_{\mathrm{pl}}^{2}}{2}\left(\frac{V_{\phi}}{V}\right)^{2}, \quad \eta=M_{\mathrm{pl}}^{2} \frac{V_{\phi \phi}}{V}
$$

are small, $\epsilon,|\eta| \ll 1$. To leading order in these parameters, each mode $Q_{k}$ satisfies the equation

$$
\ddot{Q}_{k}+3 H \dot{Q}_{k}+H^{2}\left[\frac{k^{2}}{a^{2} H^{2}}+3 \eta-6 \epsilon\right] Q_{k}=0 .
$$

Analytic solutions for Eq. (11) can be found if the slowroll parameters are constant. More generally, along the lines of $[11,12]$, we can study this equation in the infrared (IR) regime, corresponding to $k /(a H)<c$ and in the ultraviolet (UV) regime, corresponding to $k /(a H)>c$, where $1 / 10 \leqq c \lesssim 1$. We will shortly see that we can "match" the UV solution to the IR one at $k /(a H)=c$. In the UV, the slow-roll parameters can be considered as constant. The canonically normalized solution to Eq. (11) with adiabatic vacuum initial conditions then reads

$$
Q_{k}^{(\mathrm{UV})}=\frac{1}{a^{3 / 2}} \sqrt{\frac{\pi(1+\epsilon)}{4 H}} H_{\nu}^{(1)}\left[\frac{k}{a H}(1+\epsilon)\right],
$$


where $H_{\nu}^{(1)}$ is the Hankel function of the first kind with index $\nu=\frac{3}{2}-\eta+3 \epsilon$. It is instructive to rewrite Eq. (9) in the form

$$
\begin{gathered}
\left(a Q_{k}\right)^{\prime \prime}+\left(k^{2}-\frac{z^{\prime \prime}}{z}\right) a Q_{k}=0, \\
\text { where } z=a \frac{\dot{\phi}}{H}=-a M_{\mathrm{pl}} \sqrt{2 \epsilon},
\end{gathered}
$$

and primes denote derivatives with respect to conformal time $\tau$, defined by $a d \tau=d t$. Equation (13) is simply the equation of a harmonic oscillator with a negative timedependent mass $-z^{\prime \prime} / z$. When $k^{2}-\frac{z^{\prime \prime}}{z}<0$, this leads to amplification on the mode $a Q_{k}$. This form of the perturbation equation is completely general and independent of the form of the potential.

In the far IR, $v \ll 1$, one can neglect the term $k^{2}$, and the nondecaying mode of the solution $Q_{k}$ is well approximated by $Q_{k} \propto \frac{\dot{\phi}}{H}=-M_{\mathrm{pl}} \sqrt{2 \epsilon}$. On the other hand, in the far UV, $-k \tau \simeq v \gg 1$ and one can neglect the term $z^{\prime \prime} / z$, so that Eq. (13) reduces to the equation for a simple harmonic oscillator.

As mentioned above, by imposing that the UV solution approximately matches the IR solution for $k /(a H)=c$, we obtain the solution valid for $k<c a H$, namely [12]

$$
Q_{k}^{(\mathrm{IR})}=\frac{1}{a^{3 / 2}} \sqrt{\frac{\pi(1+\epsilon)}{4 H}}\left(\frac{H_{c}}{H}\right)^{\gamma} H_{3 / 2}^{(1)}\left[\frac{k}{a H}(1+\epsilon)\right] .
$$

Here, $H_{c}$ is the value of the Hubble parameter at the time $t_{c}$ when $k=c a H$, and

$$
\gamma=3+\frac{V_{\phi \phi}}{3 \dot{H}}=3\left(1-\frac{\eta}{3 \epsilon}\right) .
$$

We now turn to tensor perturbations $h_{i j}$. In Fourier space, both tensor polarizations evolve according to

$$
\ddot{h}_{k}+3 H \dot{h}_{k}+\frac{k^{2}}{a^{2}} h_{k}=0
$$

As before, one can derive approximate solutions in the UV and IR. The mode and the amplitude are chosen such that the canonically normalized variable $\left(a M_{\mathrm{pl}} / \sqrt{2}\right) h$ satisfies adiabatic vacuum initial conditions in the UV. Thus, one finds

$$
h_{k}^{(\mathrm{UV})}=\frac{1}{a^{3 / 2} M_{\mathrm{pl}}^{2}} \sqrt{\frac{\pi(1+\epsilon)}{2 H}} H_{\nu}^{(1)}\left[\frac{k}{a H}(1+\epsilon)\right],
$$

with $\nu=3 / 2+\epsilon$, and

$$
h_{k}^{(I R)}=\frac{1}{a^{3 / 2} M_{\mathrm{pl}}^{2}} \sqrt{\frac{\pi(1+\epsilon)}{2 H}}\left(\frac{H_{c}}{H}\right) H_{3 / 2}^{(1)}\left[\frac{k}{a H}(1+\epsilon)\right] \text {. }
$$

\section{B. Power spectra}

With the solutions discussed in the previous subsection, we can now compute the scalar and tensor power spectrum, defined by

$$
P_{\zeta}(k)=\frac{k^{3}}{2 \pi^{2}}\left(\frac{H}{\dot{\phi}}\right)^{2}\left|Q_{k}\right|^{2}, \quad P_{t}(k)=\frac{2 k^{3}}{\pi^{2}}\left|h_{k}\right|^{2} .
$$

In terms of the variable $v=k /(a H)$, the expansion of the spectra to first order in the slow-roll parameters, in the UV and IR, respectively, yields

$$
\begin{gathered}
P_{\zeta}^{(\mathrm{UV})}=\frac{1}{2 M_{\mathrm{pl}}^{2}}\left(\frac{H_{v}}{2 \pi}\right)^{2}\left[\frac{1+v^{2}+f(v) \epsilon_{v}+g(v) \eta_{v}}{\epsilon_{v}}\right] \\
P_{t}^{(\mathrm{UV})}=\frac{8}{M_{\mathrm{pl}}^{2}}\left(\frac{H_{v}}{2 \pi}\right)^{2}\left[1+v^{2}+f_{t}(v) \epsilon_{v}\right]
\end{gathered}
$$

and

$$
\begin{array}{r}
P_{\zeta}^{(\mathrm{IR})}=\frac{1}{2 M_{\mathrm{pl}}^{2}}\left(\frac{H_{v}}{2 \pi}\right)^{2}\left(\frac{H_{c}}{H_{v}}\right)^{2 \gamma}\left[\frac{1+v^{2}-2 \epsilon_{v}}{\epsilon_{v}}\right], \\
P_{t}^{(\mathrm{IR})}=\frac{8}{M_{\mathrm{pl}}^{2}}\left(\frac{H_{v}}{2 \pi}\right)^{2}\left(\frac{H_{c}}{H_{v}}\right)^{2}\left[1+v^{2}-2 \epsilon_{v}\right] .
\end{array}
$$

These UV and IR spectra have the correct asymptotic form, but they do not match exactly at $v=c$ since we have neglected the decaying mode contribution in $P^{(\mathrm{IR})}$. The small discontinuity is of the order of the slow-roll parameters. The functions $f(v), f_{t}(v)$, and $g(v)$ appearing in these expressions are defined and plotted in Appendix A. $H_{v}, \epsilon_{v}$, and $\eta_{v}$ are the values of these quantities calculated at the time $t_{v}$ for which $k /(a H)=v$. The functions $f(v), f_{t}(v)$, and $g(v)$ always appear multiplied by $\eta$ or $\epsilon$. Therefore, they are always subdominant for wave numbers $k$ which exit the Hubble scale in the slow-roll regime, i.e. when $\epsilon_{v} \ll 1$ and $\eta_{v} \ll 1$.

An important observable parameter is the tensor-toscalar ratio $r=P_{t} / P_{\zeta}$. On considering the particular case when $V=m^{2} \phi^{2} / 2$, we have $\eta=\epsilon$ and $\gamma=2$. Therefore, for this particular case, and at the leading order in the slow-roll parameters, we find

$$
r^{(\mathrm{UV})}=16 \epsilon_{v}, \quad r^{(\mathrm{IR})}=16 \epsilon_{c} .
$$

Note that, during slow-roll evolution, $\epsilon$ varies slowly ( $\dot{\epsilon}$ is second order in the slow-roll parameters), so that also $r^{\text {(IR) }}$ is nearly constant for scales which reach $k=c a H$ during slow roll.

To compare our findings with the five-year WMAP results, we must write $r^{(\mathrm{UV}, \mathrm{IR})}$ in terms of the spectral index $n_{s}=1+\frac{d}{d \ln k} \ln P_{\zeta}$. In turn, $n_{s}$ must be expressed as a function of $v$ and $N$, i.e. the number of $e$ folds between the epoch when the modes corresponding to the scales probed by WMAP exit the Hubble scale and the end of 
inflation. In Appendix $\mathrm{B}$, we show that when $V=$ $m^{2} \phi^{2} / 2$,

$$
n_{s}^{(\mathrm{UV})}=1-4 \epsilon_{v}, \quad n_{s}^{(\mathrm{IR})}=1-4 \epsilon_{c},
$$

where

$$
\epsilon_{s}=\eta_{s}=\frac{1}{2(N+\ln s)},
$$

while $s$ is either $v$ or $c$. It then follows that

$$
r^{(\mathrm{UV}, \mathrm{IR})}=4\left(1-n_{s}^{(\mathrm{UV}, \mathrm{IR})}\right) .
$$

The generic slow-roll expression for the scalar spectral index is [10]

$$
n_{s}=1-6 \epsilon+2 \eta .
$$

One easily verifies that for general chaotic inflation models with $V=\frac{\lambda}{p} \frac{\phi^{p}}{M_{\mathrm{pl}}^{p-4}}$, one has $\epsilon=\frac{p^{2}}{2}\left(\frac{M_{\mathrm{pl}}}{\phi}\right)^{2}$, while $\eta=p(p-1) \times$ $\left(\frac{M_{\mathrm{pl}}}{\phi}\right)^{2}$, hence

$$
n_{s}=1-\left(2+\frac{4}{p}\right) \epsilon
$$

\section{RENORMALIZED POWER SPECTRA}

We now investigate how the power spectrum is modified, when corrected by the subtraction of the adiabatic expansion up to the second order.

Let us briefly review how to obtain the adiabatic contribution to the power spectrum, in terms of the Mukhanov variable, for the scalar perturbations. As explained in the Appendix of [13], it is more convenient to formulate the adiabatic expansion by using the modulus of the Mukhanov variable $x_{k}=\sqrt{2}\left|Q_{\mathbf{k}}\right|$, which satisfies the Pinney equation

$$
\ddot{x}_{k}+3 H \dot{x}_{k}+\left[\frac{k^{2}}{a^{2}}+V_{\phi \phi}+2 \frac{d}{d t}\left(3 H+\frac{\dot{H}}{H}\right)\right] x_{k}=\frac{1}{a^{6} x_{k}^{3}} .
$$

In conformal time, the above equation simplifies to

$$
\left(a x_{k}\right)^{\prime \prime}+\Omega^{2}\left(a x_{k}\right)=\frac{1}{\left(a x_{k}\right)^{3}},
$$

where

$$
\Omega_{k}^{2}=k^{2}+a^{2} V_{\phi \phi}-\frac{1}{6} a^{2} \tilde{R},
$$

and

$$
\tilde{R}=R-6\left(-4 \frac{a^{\prime 2}}{a^{4}}-2 \frac{a^{\prime \prime 2}}{a^{2} a^{\prime 2}}+2 \frac{a^{\prime \prime \prime}}{a^{2} a^{\prime}}\right), \quad R=6 \frac{a^{\prime \prime}}{a} .
$$

From these equations, one obtains the WKB expansion for $x_{k}$ up to the second adiabatic order, which reads

$$
x_{k}=\frac{1}{a} \frac{1}{\Omega_{k}^{1 / 2}}\left(1+\frac{1}{8} \frac{\Omega_{k}^{\prime \prime}}{\Omega_{k}^{3}}-\frac{3}{16} \frac{\Omega_{k}^{\prime 2}}{\Omega_{k}^{4}}\right) \text {. }
$$

In turn, from this expression one immediately finds the second order adiabatic expansion of $\left|Q_{k}\right|^{2}$, namely

$$
\left|Q_{k}\right|^{2}=\frac{1}{2 a^{2} \Omega_{k}}\left(1+\frac{1}{4} \frac{\Omega_{k}^{\prime \prime}}{\Omega_{k}^{3}}-\frac{3}{8} \frac{\Omega_{k}^{\prime 2}}{\Omega_{k}^{4}}\right) .
$$

The term $V_{\phi \phi}$ could be considered of adiabatic order zero in $\Omega_{k}^{2}$, but by using the field equations together with Eq. (10), one obtains a different conclusion, namely, that $V_{\phi \phi}=3 H^{2} \eta$, which is in general of adiabatic order two as well as $\tilde{R}$ (see [13] for a different interpretation). Below, we briefly discuss the case when $V_{\phi \phi}$ is considered as of order zero. In terms of the slow-roll parameters we have, at leading order,

$$
\begin{gathered}
R=6 H^{2}(2-\epsilon), \quad \tilde{R}=6 H^{2}(2+5 \epsilon), \\
\Omega_{k}^{2}=k^{2}+a^{2} H^{2}(3 \eta-5 \epsilon-2) .
\end{gathered}
$$

Since the time dependence of $\Omega_{k}^{2}$ is already of second order, any derivative of $\Omega_{k}$ generates terms of adiabatic order greater than two. Thus, we can neglect the derivatives in Eq. (35), and the power spectrum to second adiabatic order is simply

$$
\begin{gathered}
P_{\zeta}^{(2)} \equiv \frac{k^{3}}{2 \pi^{2}}\left(\frac{H_{v}}{\dot{\phi}}\right) \frac{1}{2 a^{2} \Omega_{k}} \\
=\frac{1}{2 M_{\mathrm{pl}}^{2}}\left(\frac{H_{v}}{2 \pi}\right)^{2} \frac{1}{\epsilon_{v}} \frac{v^{3}}{\sqrt{\left|2-v^{2}-3 \eta_{v}+5 \epsilon_{v}\right|}},
\end{gathered}
$$

where we used the relation $\left(\dot{\phi} / H_{v}\right)^{2}=2 M_{\mathrm{pl}} \epsilon_{v}$. The absolute value is necessary because, for $v^{2}<2-3 \eta_{v}+5 \epsilon_{v} \simeq$ 2 , one obtains a negative $\Omega_{k}^{2}$. The divergence at $v^{2}=2-$ $3 \eta_{v}+5 \epsilon_{v}$ is not relevant in the far IR $v \ll 1$ or the far UV $v \gg 1$, but it indicates that one cannot trivially connect these two regions.

The standard adiabatic expansion then goes on to expand Eq. (39) again up to second order (see, for example, [13]), thus the spectrum reads

$$
P_{\zeta}^{(2)} \simeq \frac{1}{2 M_{\mathrm{pl}}}\left(\frac{H_{v}}{2 \pi}\right)^{2} \frac{1}{\epsilon_{v}}\left(1+v^{2}-\frac{3}{2} \eta_{v}+\frac{5}{2} \epsilon_{v}\right) .
$$

This expansion is clearly meaningful only in the far UV, i.e. for $v \gg 1$. If one wants to extend the validity of adiabatic renormalization to the IR, one should use Eq. (39), which, however, becomes rapidly negligible with respect to $P_{\zeta}^{(\mathrm{IR})}$, when $v \ll 1$.

The adiabatic expansion for the tensor perturbation can be found using the results in Appendix A of [14] with $m^{2}=$ 0 . Here, the authors consider a scalar field propagating on an unperturbed space-time. On such a background, the equation of motion of the scalar field with $m^{2}=0$ coincides exactly with the equation of motion of the tensor perturbation, and one obtains directly 


$$
x_{k}=\frac{1}{a \sqrt{k^{2}-\frac{1}{6} a^{2} R}},
$$

where now $x_{k}=\left|h_{k}\right| M_{\mathrm{pl}}$. Thus, the tensor adiabatic power spectrum reads

$$
P_{t}^{(2)}=\frac{8}{M_{\mathrm{pl}}^{2}}\left(\frac{H_{v}}{2 \pi}\right)^{2} \frac{v^{3}}{\sqrt{\left|2-v^{2}-\epsilon_{v}\right|}} .
$$

As above, in the far UV one can expand for large $v$, up to second abiabatic order, and find for the adiabatic spectrum

$$
P_{t}^{(2)} \simeq \frac{8}{M_{\mathrm{pl}}^{2}}\left(\frac{H_{v}}{2 \pi}\right)^{2}\left(v^{2}+1-\frac{\epsilon_{v}}{2}\right) .
$$

With similar consideration as above, one sees that $P_{t}^{(2)}$, when expressed as in Eq. (42), becomes rapidly negligible with respect to $P_{t}^{(\mathrm{IR})}$ when $v \ll 1$.

These results show that the extension to the IR of the adiabatic expansions of the scalar and tensor spectra, if sensible, must be considered with great care. In fact, in Figs. 1 and 2, one sees that the adiabatic corrections have very different effects, according to whether one uses Eqs. (39) and (42) or Eqs. (40) and (43).

We now consider the UV case in more detail. The renormalized power spectra are given by

$$
P_{\zeta}=P_{\zeta}^{(\mathrm{UV})}-P_{\zeta}^{(2)}, \quad P_{t}=P_{t}^{(\mathrm{UV})}-P_{t}^{(2)},
$$

where $P_{\zeta}^{(2)}$ and $P_{t}^{(2)}$ can be calculated using either Eqs. (40) and (43) or Eqs. (39) and (42), as these fully coincide for large $v$. However, when $v=1$, the corresponding expressions for $P_{\zeta}$ already differ substantially. In fact, to leading order in the slow-roll parameters, using (40), we find

$$
P_{\zeta} \simeq \frac{1}{2 M_{\mathrm{pl}}^{2}}\left(\frac{H}{2 \pi}\right)^{2} \frac{1}{\epsilon}\left[3 \alpha \epsilon+\left(3 \beta-\frac{9}{4}\right) \eta\right],
$$

while, with Eq. (39), we obtain

$$
P_{\zeta} \simeq \frac{1}{2 M_{\mathrm{pl}}^{2}}\left(\frac{H}{2 \pi}\right)^{2} \frac{1}{\epsilon}\left[1+(3 \alpha+5) \epsilon+\left(3 \beta-\frac{21}{4}\right) \eta\right],
$$

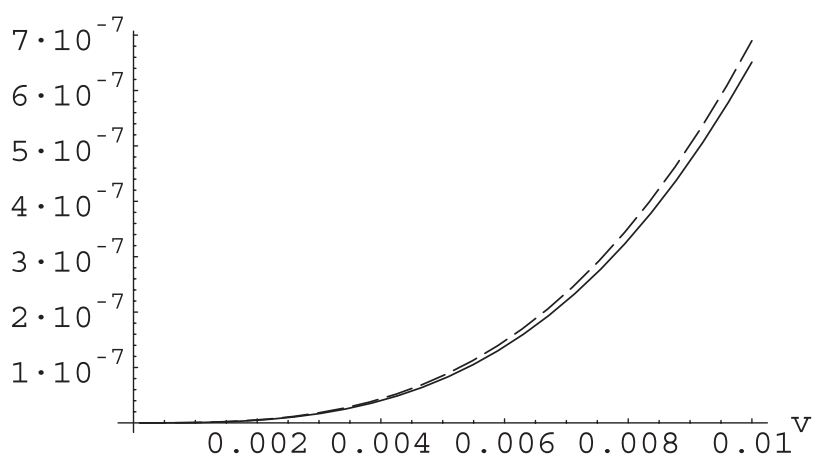

FIG. 1. The ratios $P_{\zeta}^{(2)} / P_{\zeta}$ (solid line) and $P_{t}^{(2)} / P_{t}$ (dashed line) in the IR, $v \ll 1$, calculated with Eqs. (39) and (42), for the case $V=m^{2} \phi^{2} / 2$. These contributions are negligible for both scalar and tensor perturbations. where $\alpha \simeq 0.903, \beta \simeq 0.449$, and all of the parameters are calculated at the time when $k=a H$. These results make it clear that adiabatic counterterms, which agree in the far UV and correctly renormalize the theory in this regime, produce different results not only in the far IR, but also at horizon exit. Similar considerations hold for the tensor spectrum.

To better compare our results with the ones in [7], we now discuss the case where one considers $V_{\phi \phi}$ in the scalar spectrum of adiabatic order zero and $\tilde{R}$ of order two. Starting from Eq. (35) and expanding again up to second order, one obtains

$$
\begin{aligned}
P_{\zeta}^{(2)}= & \frac{1}{2 M_{\mathrm{pl}}^{2}}\left(\frac{H_{v}}{2 \pi}\right)^{2} \frac{1}{\epsilon_{v}}\left[\frac{v^{3}}{\left(v^{2}+3 \eta_{v}\right)^{1 / 2}}+\frac{v^{3}\left(1+\frac{5}{2} \epsilon_{v}\right)}{\left(v^{2}+3 \eta_{v}\right)^{3 / 2}}\right. \\
& \left.+\frac{9 \eta_{v} v^{3}}{4\left(v^{2}+3 \eta_{v}\right)^{5 / 2}}-\frac{45 \eta_{v}^{2} v^{3}}{8\left(v^{2}+3 \eta_{v}\right)^{7 / 2}}\right]
\end{aligned}
$$

where we have kept only the leading order, in the slow-roll parameters, in each term. With this expression, the renormalized $P_{\zeta}$ differs again from the other two expressions above. To see this, we set $v=1$ and expand with respect to the slow-roll parameters to find the expression

$$
P_{\zeta}=\frac{1}{2 M_{\mathrm{pl}}^{2}}\left(\frac{H}{2 \pi}\right)^{2} \frac{1}{\epsilon}(3 \alpha \epsilon+3 \beta \eta)
$$

which has to be compared to Eq. (10) of [7]. In this paper, there is just $\alpha$ instead of $3 \alpha$. This comes from the fact that we use the Mukhanov variable, while in [7] the authors use the scalar inflaton perturbation in a space-time without metric fluctuations. However, when $v \ll 1$, this result is no longer reliable as we simply cannot expand with respect to the slow-roll parameter, since $3 \eta_{v}$ is no longer much smaller than $v^{2}$. For example, in the case $V=m^{2} \phi^{2} / 2$, as shown in Appendix B, we have $3 \epsilon_{v} \equiv 3 \eta_{v} \simeq 1 /(2 N)$. So, if $N=50$ and $v=1 / 5$, then $v^{2}=1 / 25$ and $3 \eta_{v}=$ $3 / 100$. As a result of this, the tensor-to-scalar ratio strongly depends on $v$ for $v<1$.

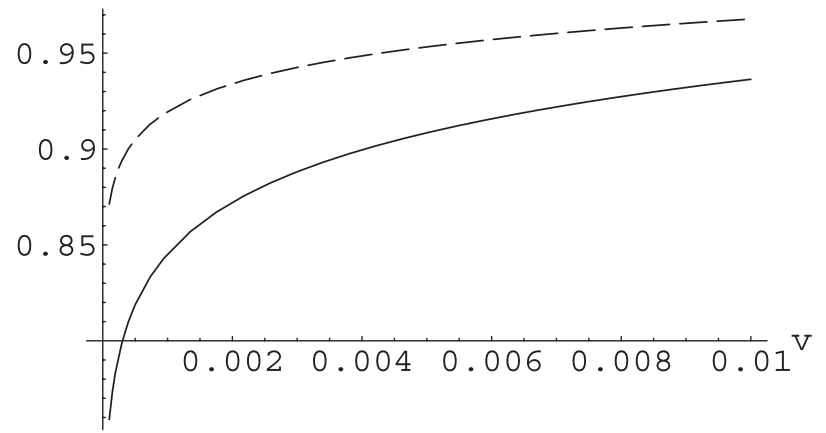

FIG. 2. The ratios $P_{\zeta}^{(2)} / P_{\zeta}$ (solid line) and $P_{t}^{(2)} / P_{t}$ (dashed line) in the IR, $v \ll 1$, calculated with Eqs. (40) and (43), for the case $V=m^{2} \phi^{2} / 2$. These contributions are of considerable size for a large range of $v$, however they are not correct as in this regime, the expansions (40) and (43) are not valid. 
In addition to the above considerations, it is known that the long wave adiabatic modes do not contribute significantly to the renormalized Green function at coincident points in realistic inflationary models (see [15] for similar consideration on massless particle).

The main point of this section is that adiabatic subtraction is not a reliable technique in the IR. In fact, we have presented three types of counterterms for the scalar spectrum, Eqs. (39), (40), and (47), and two for the tensor one, namely, Eqs. (42) and (43). In each case, these terms are equivalent in the far UV but give very different results elsewhere. Furthermore, we have shown that when using (39) and (42), the subtracted spectra are close to the bare ones already at $v=1$ and virtually identical to them at $v \ll 1$.

\section{CONCLUSIONS}

In this paper, we have first determined the renormalized perturbation spectra in a slow-roll inflationary model in UV domain. In agreement with Ref. [7], we have found that adiabatic subtraction can lead to a substantial reduction of power in the UV. Namely, the larger $k / a$ in comparison to $H$, the closer the weight of the adiabatic counterterm becomes to the unrenormalized spectra. This is reasonable, since we do not expect that the expansion of the Universe is "energetic enough" to excite physical modes in the UV.

On the contrary, in the IR, the adiabatic expansion is no longer valid, and there is no convincing physical argument to subtract this term to the standard power spectrum. As there is a natural IR cutoff to inflation, we propose that for cosmologically relevant scales, which have been amplified by inflation but are in the far IR at the end of inflation, no adiabatic subtraction should be performed.

One might argue, however, that this expansion scheme still produces a finite result and therefore provides a way to renormalize the IR modes. In fact, we find this argument not convincing, as there are different schemes to renormalize the IR modes (for example, see the recent paper [16]). In a way, we have also shown this by presenting different counterterms which agree in the far UV but not in the IR. This reflects the well-known result that in the far UV, where space-time curvature becomes negligible, the physical spectrum is independent of the regularization scheme and that the UV singularity structure of the two-point function is always of the Hadamard form. Furthermore, since inflation has not started in the infinite past, there is a natural infrared cutoff, namely, the horizon scale at the beginning of inflation. We therefore conclude that one should not subtract the adiabatic contribution in the IR in realistic inflationary models.

Even though the adiabatic calculation does not apply in the IR, it is interesting to note that, the adiabatic counterterms become much smaller than the unrenormalized spectrum in the IR, when computed without any expansion, as in Eqs. (39) and (42). As mentioned above, these expressions show a singularity for $v^{2} \simeq 2$. This is not relevant, from our point of view, as we claim that the subtraction should be performed at the end of inflation. At the end of inflation, however, all cosmologically relevant scales are in the far IR, hence the adiabatic subtraction, which is a possible prescription, since the far UV does not affect the associated spectra. This is the main conclusion of this work.

The adiabatic subtraction does, however, provide a clean means to derive the shape of the physical spectrum in the UV, where it actually tends to zero: at any given time, fluctuations with $v>1$ are significantly suppressed by the adiabatic counterterm. In this sense, the adiabatic subtraction provides a UV cutoff of the spectrum which is roughly given by the scale $k_{\mathrm{UV}}$ which reaches $v=1$ at the end of inflation $k_{\mathrm{UV}}=a_{f} H_{f}$.

The reason why it is usually sensible to compute $P_{\zeta}$ and $P_{t}$ at the Hubble exit $v \simeq 1$ instead of evaluating them at the end of inflation, is that we have simple and sound formulae for them, which are valid "inside the Hubble scale," while the growing modes of the perturbations are nearly constant "outside the Hubble scale." Therefore, in general we do not need to calculate their evolution until the end of inflation. This is different for the adiabatic counterterm $P^{(2)}$ : as we have shown, this term becomes strongly time dependent and decreases with $v$ in the IR, if extended in this regime without any further expansion. Therefore, it seems reasonable to perform the adiabatic subtraction at the end of inflation, or at least far in the IR, where, however, it becomes irrelevant for all scales of cosmological interest.

\section{ACKNOWLEDGMENTS}

We thank Ivan Agulló, José Navarro-Salas, Gonzalo J. Olmo, and Leonard Parker for interesting and clarifying discussions. G. M. also thanks Alexei A. Starobinsky for useful discussions. This work is supported by the Swiss National Science Foundation.

\section{APPENDIX A: DEFINITIONS}

In Sec. II B, we present the renormalized power spectrum of curvature and tensor perturbations, expanded with respect to the slow-roll parameters. The three functions $f$, $g$, and $f_{t}$ appearing in these expressions are defined by

$$
\begin{aligned}
f(v)= & -2-3 \sqrt{2 \pi} v^{5 / 2}\left\{\cos (v)\left[\frac{\partial}{\partial \nu} J_{\nu}(v)\right]_{\nu=3 / 2}+\sin (v)\right. \\
& \left.\times\left[\frac{\partial}{\partial \nu} J_{\nu}(v)\right]_{\nu=3 / 2}\right\}+3 \sqrt{2 \pi} v^{3 / 2}\{\sin (v) \\
& \left.\times\left[\frac{\partial}{\partial \nu} J_{\nu}(v)\right]_{\nu=3 / 2}-\cos (v)\left[\frac{\partial}{\partial \nu} J_{\nu}(v)\right]_{\nu=3 / 2}\right\}
\end{aligned}
$$




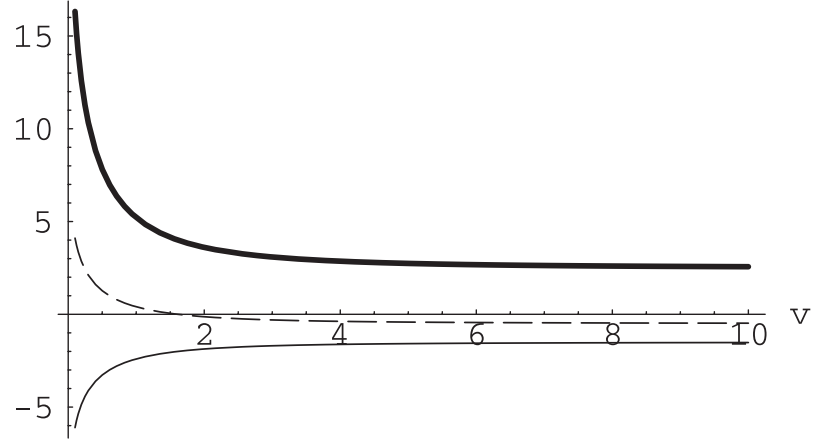

FIG. 3. Plots of the behavior of $f$ (thick solid line), $g$ (thin solid line), and $f_{t}$ (dashed line) in the function of $v=k /(a H)$ in the range from $1 / 10$ to 10 .

$$
\begin{aligned}
g(v)= & \sqrt{2 \pi} v^{5 / 2}\left\{\cos (v)\left[\frac{\partial}{\partial \nu} J_{\nu}(v)\right]_{\nu=3 / 2}+\sin (v)\right. \\
& \left.\times\left[\frac{\partial}{\partial \nu} J_{\nu}(v)\right]_{\nu=3 / 2}\right\}-\sqrt{2 \pi} v^{3 / 2}\{\sin (v) \\
& \left.\times\left[\frac{\partial}{\partial \nu} J_{\nu}(v)\right]_{\nu=3 / 2}-\cos (v)\left[\frac{\partial}{\partial \nu} J_{\nu}(v)\right]_{\nu=3 / 2}\right\} \\
f_{t}(v)= & -2-\sqrt{2 \pi} v^{5 / 2}\left\{\cos (v)\left[\frac{\partial}{\partial \nu} J_{\nu}(v)\right]_{\nu=3 / 2}+\sin (v)\right. \\
& \left.\times\left[\frac{\partial}{\partial \nu} J_{\nu}(v)\right]_{\nu=3 / 2}\right\}+\sqrt{2 \pi} v^{3 / 2}\{\sin (v) \\
& \left.\times\left[\frac{\partial}{\partial \nu} J_{\nu}(v)\right]_{\nu=3 / 2}-\cos (v)\left[\frac{\partial}{\partial \nu} J_{\nu}(v)\right]_{\nu=3 / 2}\right\}
\end{aligned}
$$

These functions are plotted in the range $1 / 10<v<10$ in Fig. 3.

\section{APPENDIX B: SPECTRAL INDICES}

We consider the case $V=m^{2} \phi^{2} / 2$. During slow roll, we can neglect the terms $\dot{\phi}^{2}$ in Eq. (3) and $\ddot{\phi}$ in Eq. (2). It follows that $H \simeq H_{i}+\dot{H} t$, where $H_{i}$ is the initial value of the Hubble factor, and $\dot{H} \simeq-m^{2} / 3$. Thus, the scale factor satisfies the equalities

$$
\ln \frac{a(t)}{a_{i}}=\left(H_{i} t-\frac{m^{2}}{6} t^{2}\right)=\frac{3}{2 m^{2}}\left[H_{i}^{2}-H^{2}(t)\right],
$$

where $a_{i}$ is its initial value. If we assume that inflation finishes approximately when $H(t) \simeq 0$, it follows that

$$
N \equiv \ln \frac{a_{f}}{a_{N}}=\frac{3}{2} \frac{H_{N}^{2}}{m^{2}}, \quad a_{N}=e^{-N} a_{f},
$$

where $H_{N}$ and $a_{N}$ are the values of the Hubble and scale factors $N e$ folds before the end of inflation. Thus, we can write the momentum $k_{N}$, associated to the mode that exits the Hubble scale at $N e$ folds before the end of the inflation, as

$$
k_{N} \equiv a_{N} H_{N}=m\left(\frac{2}{3} N\right)^{1 / 2} a_{i} \exp \left(\frac{3 H_{i}^{2}}{2 m^{2}}-N\right) .
$$

Let $s$ be $v$ or $c$, according to whether we are dealing with the UV or IR, respectively. Let $t_{s}$ be the time when

$$
k_{N}=s a\left(t_{s}\right) H\left(t_{s}\right),
$$

and with the help of Eqs. (B1) and (B3), we find a quadratic equation in $t_{s}$, which gives

$$
t_{s}=\frac{3 H_{i}}{m^{2}}-\frac{\sqrt{6}}{m} \sqrt{N+\ln s},
$$

where we have replaced the slowly varying function $H\left(t_{s}\right)$ with the constant value $H_{N}$ in order to obtain an analytical solution of Eq. (B4). Then, as $H=H_{i}-m^{2} t / 3$, we find

$$
H\left(t_{s}\right)=m \sqrt{\frac{2}{3}(N+\ln s)},
$$

from which it follows that

$$
\epsilon_{s}=\eta_{s}=2\left(\frac{M_{\mathrm{pl}}}{\phi_{s}}\right)^{2}=\frac{m^{2}}{3 H_{s}^{2}}=\frac{1}{2(N+\ln s)} .
$$

A possible way to evaluate $n_{s}$ is to express, at leading order, the derivative with respect to $\ln k$ as

$$
\frac{d}{d \ln k} \simeq-\frac{d}{d N},
$$

which yields, in both regimes,

$$
n_{s}^{(\mathrm{UV})}=1-4 \epsilon_{v}, \quad n_{s}^{(\mathrm{IR})}=1-4 \epsilon_{c} .
$$

In the UV, we obtain the standard result, while in the IR we have a slightly different expression. In fact, even if the relation between $r$ and $n_{s}$ is the same in the two regimes, namely $r=4-4 n_{s}$, in the IR we have $n_{s}=1-2 /(N+$ $\ln c)$, while in the UV we have $n_{s}=1-2 /(N+\ln v)$. This difference is, however, quite small since $N \gg 1$ for scales which exit during slow roll.
[1] V.F. Mukhanov and G. V. Chibisov, JETP Lett. 33, 532 (1981) [Pis'ma Zh. Eksp. Teor. Fiz. 33, 549 (1981)]; V.
Mukhanov, H. Feldman, and R. Brandenberger, Phys. Rep. 215, 203 (1992). 
[2] A. Starobinsky, JETP Lett. 30, 682 (1979) [Pis'ma Zh. Eksp. Teor. Fiz. 30, 719 (1979)].

[3] G. Hinshaw et al., Astrophys. J. Suppl. Ser. 180, 225 (2009); M. R. Nolte et al., Astrophys. J. Suppl. Ser. 180, 296 (2009); J. Dunkley et al., Astrophys. J. Suppl. Ser. 180, 306 (2009).

[4] E. Komatsu et al., Astrophys. J. Suppl. Ser. 180, 330 (2009).

[5] L. Parker, arXiv:hep-th/0702216.

[6] I. Agulló, J. Navarro-Salas, G. J. Olmo, and L. Parker, Phys. Rev. Lett. 101, 171301 (2008).

[7] I. Agulló, J. Navarro-Salas, G. J. Olmo, and L. Parker, Phys. Rev. Lett. 103, 061301 (2009).

[8] F. Finelli, G. Marozzi, G. P. Vacca, and G. Venturi, Phys. Rev. D 76, 103528 (2007).

[9] V.F. Mukhanov, JETP Lett. 41, 493 (1985) [Pis'ma Zh.
Eksp. Teor. Fiz. 41, 402 (1985)]; Sov. Phys. JETP 67, 1297 (1988) [Zh. Eksp. Teor. Fiz. 94N7, 1 (1988)].

[10] R. Durrer, The Cosmic Microwave Background (Cambridge University Press, Cambridge, United Kingdom, 2008).

[11] F. Finelli, G. Marozzi, G. P. Vacca, and G. Venturi, Phys. Rev. D 69, 123508 (2004).

[12] F. Finelli, G. Marozzi, A. A. Starobinsky, G. P. Vacca, and G. Venturi, Phys. Rev. D 79, 044007 (2009).

[13] G. Marozzi, Phys. Rev. D 76, 043504 (2007).

[14] F. Finelli, G. Marozzi, G. P. Vacca, and G. Venturi, Phys. Rev. D 65, 103521 (2002).

[15] C. Kiefer, I. Lohmar, D. Polarski, and A. A. Starobinsky, Classical Quantum Gravity 24, 1699 (2007).

[16] T. M. Janssen and T. Prokopec, arXiv:0906.0666. 